\title{
Antidiabetic and Nephroprotective Effects of Polysaccharide Extract from the Seaweed Caulerpa racemosa in High Fructose-Streptozotocin Induced Diabetic Nephropathy
}

\author{
Meng Cao' \\ Yan Li' \\ Ademola C Famurewa ${ }^{2}$ \\ Opeyemi Joshua Olatunji (iD) ${ }^{3}$ \\ 'Department of Endocrinology, The First \\ Affiliated Hospital of Xinxiang Medical \\ College, Xinxiang, Henan Province, \\ People's Republic of China; ${ }^{2}$ Department \\ of Medical Biochemistry, Faculty of Basic \\ Medical Sciences, College of Medicine, \\ Alex Ekwueme Federal University, \\ Ndufu-Alike, Ebonyi State, Nigeria; \\ ${ }^{3}$ Faculty of Thai Traditional Medicine, \\ Prince of Songkla University, Hat Yai, \\ Thailand
}

Background: Nephropathy is a frontline complication of diabetes mellitus (DM) associated with impaired redox-inflammatory networks. The study investigated the antidiabetic and nephroprotective potentials of PCR against diabetic nephropathy (DN) in rats.

Methods: DN was induced in rats using a combination of a high fructose solution for 4 weeks and an intraperitoneal injection of streptozotocin $(35 \mathrm{mg} / \mathrm{kg})$. Diabetic rats were treated with PCR (100 and $400 \mathrm{mg} / \mathrm{kg}$ body weight) for 8 weeks. Serum biochemical parameters as well as renal oxidative stress parameters, proinflammatory cytokines, Western blot and histopathological analyses were evaluated.

Results: There were significant increases in fasting blood glucose, urinary albumin, serum creatinine, blood urea nitrogen (BUN), total cholesterol (TC), triglycerides (TG), and lowdensity lipoproteins (LDL-C) levels in diabetic rats compared to the non-diabetic control rats. DM-induced DN prominently depressed renal superoxide dismutase (SOD), catalase (CAT) and glutathione peroxidase (GSH-Px) activities, whereas renal malondialdehyde (MDA) level was markedly increased. Furthermore, renal inflammatory cytokines, IL-1 $\beta$, IL-6, TNF- $\alpha$ and TGF- $\beta$, were considerably elevated compared to non-diabetic control rats. Additionally, DN rats showed a significant increase in renal fibrosis, as evidenced by increased expression of TGF- $\beta 1$, collagen- 1 , fibronectin and alpha-smooth muscle actin ( $\alpha$ SMA) in the kidneys. Histopathological lesions were consistent with tubule thickening and glomerular hypertrophy. Conversely, PCR treatment exerted significant attenuation of hyperglycemia, dyslipidemia and renal oxidative stress indicators. The increased renal levels of IL$1 \beta$, IL- 6 , TNF- $\alpha$ and TGF- $\beta$ were also notably reversed dose-dependently with alleviation of nephropathic histology. Furthermore, PCR reduced the expression of $\alpha$-SMA, fibronectin, collagen-1 and TGF- $\beta 1$ in the renal tissues.

Conclusion: Our results suggest that PCR displayed antidiabetic and nephroprotective effects against DN by impeding oxidative stress and inflammation. As such, PCR has potentials as a food supplement for alleviating renal dysfunction caused by diabetes.

Keywords: diabetes, nephropathy, hyperglycemia, Caulerpa racemosa, polysaccharides

\section{Introduction}

Correspondence: Opeyemi Joshua Olatunji

Faculty of Thai Traditional Medicine,

Prince of Songkla University, Hat Yai,

90II0, Thailand

Email opeyemi.j@psu.ac.th
Diabetes mellitus (DM) is the fourth leading chronic, non-communicable disorder that accounted for almost 4.2 million mortality in 2019. The number of diabetic patients is projected to increase from 463 million in 2019 to 700 million by 2045 . $^{1,2}$ $\mathrm{DM}$ is associated with several deteriorating complications including neuropathy, 
nephropathy, retinopathy, cardiovascular diseases amongst several others, which significantly affects the quality of life of diabetic patients. ${ }^{3,4}$ Diabetic nephropathy (DN) is one of the leading and most frequently encountered complication in DM and it significantly contributes to the mortality rates in diabetic patients. ${ }^{5,6}$ The clinical features characterizing DN include thickening of the basement membrane, extracellular matrix accumulation, as well as tubular and glomerular hypertrophy. ${ }^{7,8}$ A huge number of evidence from previous studies have linked the abnormal pathological changes associated with $\mathrm{DN}$ to an intricate interplay between several factors, mainly hyperglycemiainduced oxidative stress, proinflammatory cytokines and advanced glycation-end product formation. ${ }^{9-11}$ Although there are numerous medications approved for treating diabetes, unfortunately these drugs have not shown effectiveness against $\mathrm{DN}$, as such exploring alternative effective remedies has become a major priority in recent years. Since recent mechanistic studies have implicated oxidative stress and inflammation in the pathogenesis of many diseases including metabolic disorders, suppressing hyperglycemia-induced oxidative stress, which is the major culprit implicated in DN by antioxidants, may be a reasonable approach to effectively combat DN. ${ }^{11}$

Accumulating studies have continued to showcase the importance of marine organisms, such as seaweeds as food and as sources of bioactive agents in the fight against many diseases. Seaweeds have been reported as active agents against inflammation, cancer, diabetes, bacterial infections and obesity to mention a few. ${ }^{12,13}$ Most of the reported bioactivities have been attributed to the bioactive constituents, such as proteins, polysaccharides, alkaloids, terpenoids and tannins present in the seaweeds. ${ }^{12}$ Caulerpa racemosa (sea grapes) is a species of green seaweed belonging to the family Caulerpaceae. C. racemosa is widely distributed in tropical regions especially in Indo-Pacific Asia and it is widely consumed in its raw form as salad or cooked as part of the ingredients in some vegetable soup. ${ }^{14,15}$ Numerous bioactivities have been attributed to polysaccharides or polysaccharide extracts from C. racemosa including antioxidant, antibacterial, anti-herpetic, antinociceptive and anti-inflammatory effects. $^{16-18}$ Although polysaccharides from C. racemosa have been reported to be effective in several biological assays, the kidney protective effects in diabetic models, especially DN, are yet to be explored. As such, this present study investigated the effects of the crude polysaccharide extract from C. racemosa (PCR) against DN in high fructose and streptozotocin (STZ)-induced diabetic rats.

\section{Materials and Methods Biological Material Collection and Preparation}

C. racemosa was obtained from the coastal area of Southern Thailand in May 2019. The samples were washed with running tap water to remove debris, epiphytes and residual salt. The crude polysaccharide was extracted from the freeze-dried powdered sample by hot water extraction. $100 \mathrm{~g}$ of the sample was refluxed in $1000 \mathrm{~mL}$ of distilled water thrice at $100^{\circ} \mathrm{C}$ for 2 hours. The combined water extracts were filtered and concentrated to a specific volume (approximately $10 \%$ of the original volume) with a rotary evaporator. The resulting solution was subjected to deproteinization using Sevag method and further dialysed against distilled water for 48 h. $^{19,20}$ The dialysate was freeze-dried to yield the crude polysaccharide (PCR) extract and stored at $4^{\circ} \mathrm{C}$ until further use.

\section{Animals and Treatments}

All the animal experimental protocols employed in this study received thorough review and approval from the Animal Ethics Committee of The First Affiliated Hospital of Xinxiang Medical College (Ethics approval number: 2021001). Furthermore, all procedures used were in accordance with the regulations of the National Institute of Health guide for the care and use of laboratory animals (NIH Publication No. 80-23; revised 1978). Healthy male six-week-old Wistar albino rats (140-180 g) were used in the study. Prior to the commencement of the study, the animals were housed in a facility with the following conditions: temperature (24 $\left.\pm 2{ }^{\circ} \mathrm{C}\right)$, relative humidity $(55 \pm 10 \%)$ and a $12 \mathrm{~h}$ lightdark cycle, in addition to unrestricted access to standard chow and normal tap water. After one week of acclimatization, the rats were divided into two groups: the first group comprising of six rats received standard chow and normal drinking water ad libitum, while the other group comprising of 24 rats were administered with $30 \%$ fructose solution as drinking water and standard rat chow for four weeks to induce insulin resistance. ${ }^{21,22}$ After four weeks, type $2 \mathrm{DN}$ was established in overnight fasted rats using intraperitoneal injection of STZ (35 mg/kg) dissolved in sodium citrate buffer ( $\mathrm{pH} 4.5)$. 
Three days after STZ injection, fasting glucose concentration in the blood obtained from the tail of the rats was measured using an Accu-Chek Active glucose meter (Roche Diagnostic, Germany). Rats having fasting blood glucose levels above $11.1 \mathrm{mmol} / \mathrm{L}$ were adjudged as having diabetes and were further divided into diabetic and PCR-treated groups as follows:

Normal control group (Ctrl): administered with normal saline for 8 weeks

DN control group (DNC): administered with normal saline for 8 weeks

DN+PCR-1 group: administered with $100 \mathrm{mg} / \mathrm{kg}$ body weight of PCR for 8 weeks

DN+PCR-2 group: administered with $400 \mathrm{mg} / \mathrm{kg}$ body weight of PCR for 8 weeks

The choice of the dose of PCR as well as the duration of treatment was based on our preliminary investigation supported by previous studies. ${ }^{23-26}$ After treatment for 8 weeks, the $24 \mathrm{~h}$ urine of the rats were collected for the estimation of urinary albumin. Thereafter, all the rats were fasted overnight, sacrificed, blood samples and the kidney were obtained for various biochemical and histopathological analyses. The serum concentrations of creatinine, blood urea nitrogen, cholesterol, triglycerides and low density lipoprotein cholesterol were determined with the aid of an automated biochemical analyser (Dirui CS 600B, Japan). Serum insulin (CSB-E05070r) was determined with enzymelinked immunosorbent (ELISA) from Cusabio Biotech Co., Ltd. (Hubei, China) following the manufacturer's protocol. Kidney tissue homogenates were prepared in phosphate buffered saline and used for detecting levels of IL-6 (mL-68044), IL-1 $\beta$ (mL-68035), TNF- $\alpha$ (mL31189) and TGF- $\beta$ (mL-31133) by enzyme-linked immunosorbent assay (ELISA) kits (Mlbio, Shanghai Enzyme-linked Biotechnology Co., Ltd, China). Kidney homogenates were also used for estimating hepatic levels of lipid peroxidation (MDA), superoxide dismutase (SOD), catalase (CAT) and glutathione oxidase (GSH-Px) using biochemical assay kits (Nanjing Jiancheng Bioengineering Institute, China).

\section{Histopathological Evaluations}

A small part of the kidney tissue from the left kidney was fixed in $10 \%$ buffered formalin and subjected to dehydration with a graded alcohol solution and further embedded in paraffin to make 5- $\mu \mathrm{m}$ sections. The kidney tissue sections were finally stained with hematoxylin-eosin and visualized for histopathological changes under a light microscope (Olympus DP73, Japan).

\section{Western Blot Analyses}

Relative protein expressions in the kidney tissues were analysed by Western blot. Briefly, renal tissues were lysed in RIPA buffer and further centrifuged at $15,000 \mathrm{rpm}$ for $30 \mathrm{mins}$ at $4^{\circ} \mathrm{C}$. The concentration of the proteins in the supernatant was estimated using BCA assay and samples with equal amount of proteins were loaded on SDS-PAGE and the separated proteins were further transferred to PVDF membrane and blocked with $5 \%$ bovine serum albumin. The membranes were successively incubated with primary and the corresponding secondary antibodies at $4^{\circ} \mathrm{C}$. The protein bands were visualized using ECL method via a Tanon 5200 imaging system. Semiquantification analyses of the bands were performed with Image J software.

\section{Statistical Data Analysis}

One-way ANOVA followed by Newman-Keuls multiple comparison test was utilized for data analysis using Graph Pad Prism software (version 5.0; GraphPad Software, USA). Results are shown as mean $\pm S D(n=6) . P<0.05$ was regarded as statistical significance.

\section{Results}

\section{Treatment with PCR Improves Metabolic and Renal Function Indicators in DN Rats}

As indicated in Figure 1, DNC rats showed excessive increase in their fasting blood glucose concentration, which was accompanied by significant decrease in body weight and serum insulin levels when compared to the healthy normal control group (Figure 1A-C). In addition, DNC group showed significantly increased kidney weight and kidney index compared to the normal control group (Figure 1D and E). In the PCR (100 and $400 \mathrm{mg} / \mathrm{kg}$ ) treated rats, significant reduction in the FBG concentration, kidney weight and kidney index were observed compared to the DNC group. PCR also markedly increased the body weight gain and serum insulin levels in the treated DN rat groups (Figure 1A-E). Moreover, the levels of serum creatinine, blood urea nitrogen and urinary albumin in the DN control group were significantly increased compared to the normal control, while the excessive increase in serum 

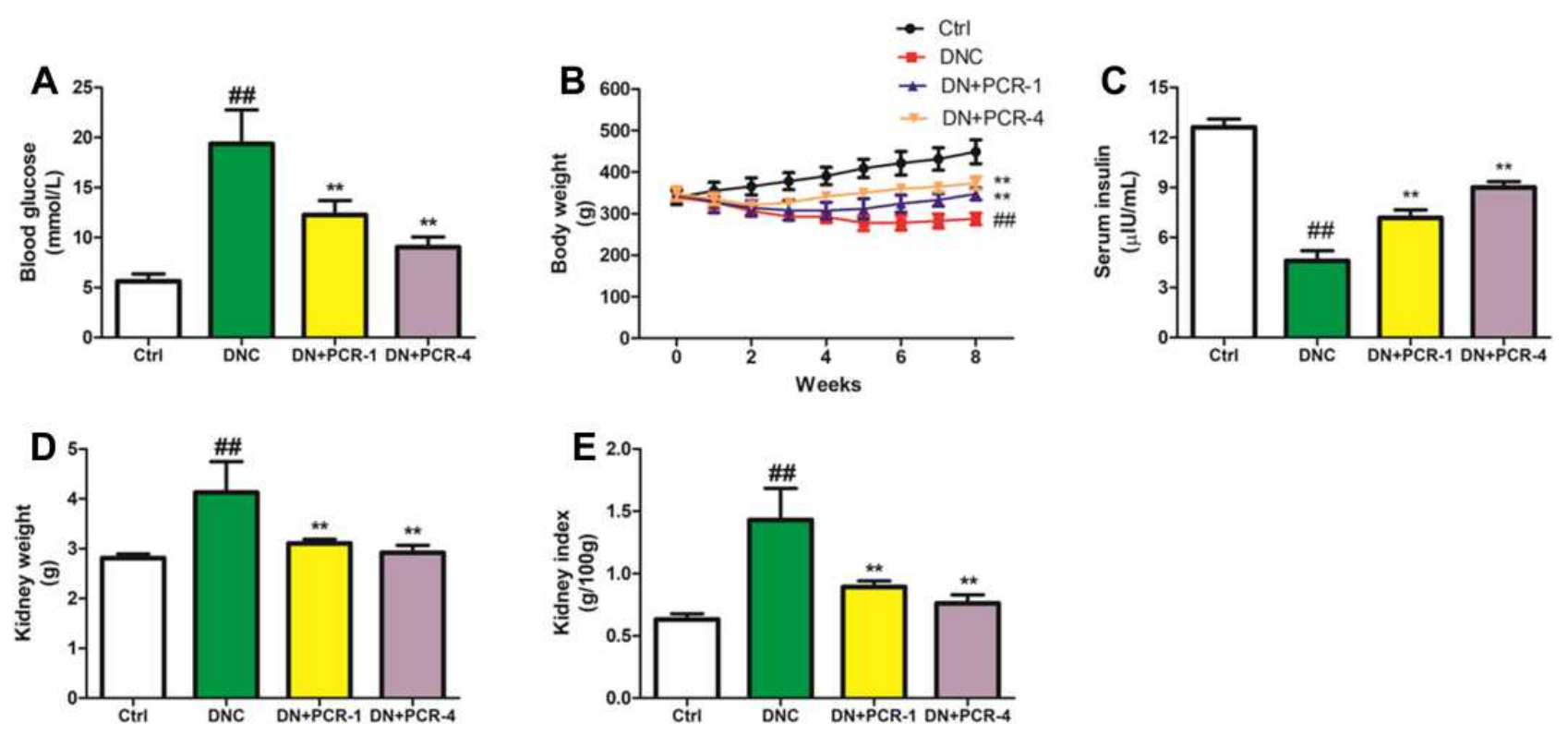

Figure I Effect of PCR on (A) fasting blood glucose concentration, (B) body weight gain, (C) serum insulin (D) kidney weight, (E) kidney index of DN rats. Data were expressed as the AQ3 means \pm SD $(n=6)$. Statistical analysis was performed by one-way ANOVA followed by Newman-Keuls post hoc test for multiple comparisons. Notes: ${ }^{\# \#} p<0.05$ vs Ctrl group; ${ }^{* *} p<0.05$ vs DNC group.

Abbreviations: Ctrl, control group; DNC, diabetic nephropathy control group; DN+PCR-I, diabetic nephropathy rats treated with PCR at I00mg/kg; DN+PCR-4, diabetic nephropathy rats treated with PCR at $400 \mathrm{mg} / \mathrm{kg}$.
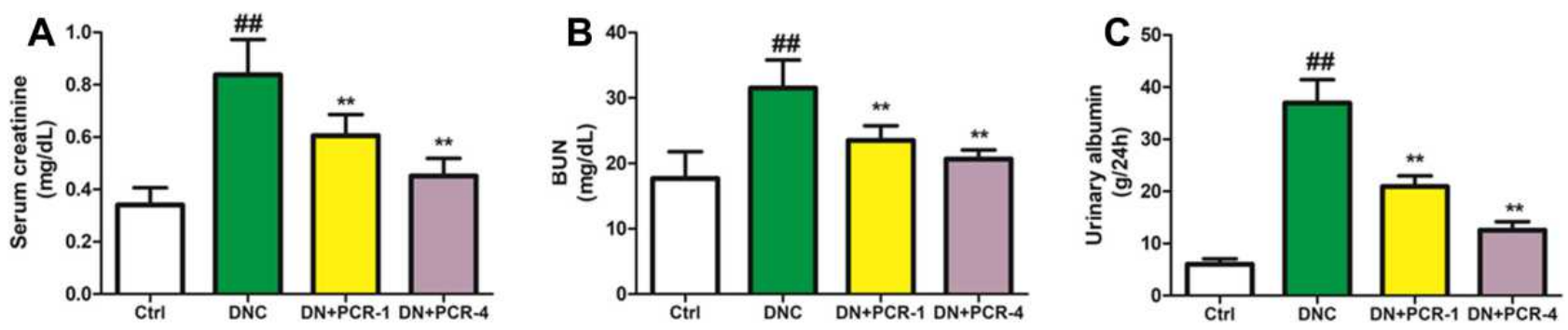

Figure 2 Effect of PCR on $(\mathbf{A})$ serum creatinine, $(\mathbf{B})$ blood urea nitrogen, $(\mathbf{C})$ urinary albumin levels in DN rats. Data were expressed as the means \pm SD $(n=6)$. Statistical analysis was performed by one-way ANOVA followed by Newman-Keuls post hoc test for multiple comparisons.

Notes: ${ }^{\#} p<0.05$ vs Ctrl group; ${ }^{* *} p<0.05$ vs DNC group.

Abbreviations: Ctrl, control group; DNC, diabetic nephropathy control group; DN+PCR-I, diabetic nephropathy rats treated with PCR at I00mg/kg; DN+PCR-4, diabetic nephropathy rats treated with PCR at $400 \mathrm{mg} / \mathrm{kg}$.

creatinine, blood urea nitrogen and urinary albumin levels in DN rats were reversed after treatment with PCR (100 and $400 \mathrm{mg} / \mathrm{kg}$; Figure 2A-C).

\section{Treatment with PCR Ameliorated Serum Biochemical Parameters in DN Rats}

As indicated in Figure 3, the serum biochemical parameters including TG, TC and LDL-C of the DN control group were significantly increased compared to those of the normal control rats (Figure 3A-C). However, in the $\mathrm{DN}$ rats that received PCR, there was a vivid decrease in these biochemical parameters compared to the DN control group.

\section{Treatment with PCR Attenuated Kidney Lipid Peroxidation and Antioxidant Enzymes}

The efficacy of PCR administration on lipid peroxidation (MDA level) in the kidney tissues of DN rats is shown in Figure 4A. The results indicated that MDA level was significantly upregulated in the DN control group compared to healthy normal control. As envisaged, the increase in MDA values was significantly reversed in response to PCR treatment. In addition, DN control rats showed a gross decrease in the activities of kidney antioxidant enzymes (CAT, SOD and GSHPx) compared to the normal control (Figure 4B-D). In 

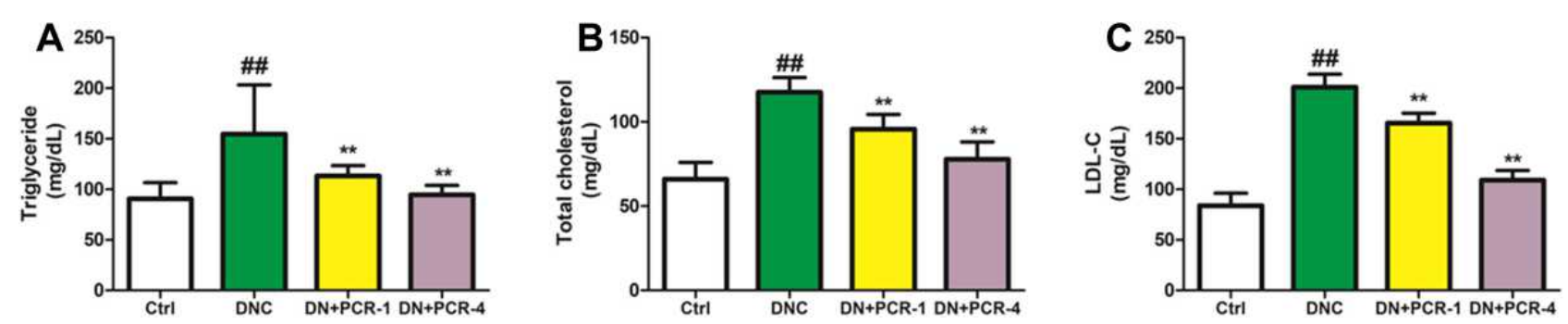

Figure 3 Effect of PCR on serum (A) triglycerides, $(\mathbf{B})$ total cholesterol, $(\mathbf{C})$ low-density lipoprotein cholesterol levels in DN rats. Data were expressed as the means \pm SD $(n=6)$. Statistical analysis was performed by one-way ANOVA followed by Newman-Keuls post hoc test for multiple comparisons.

Notes: ${ }^{\#} p<0.05$ vs Ctrl group; ${ }^{* *} p<0.05$ vs DNC group.

Abbreviations: Ctrl, control group; DNC, diabetic nephropathy control group; DN+PCR-I, diabetic nephropathy rats treated with PCR at I00mg/kg; DN+PCR-4, diabetic nephropathy rats treated with PCR at $400 \mathrm{mg} / \mathrm{kg}$.
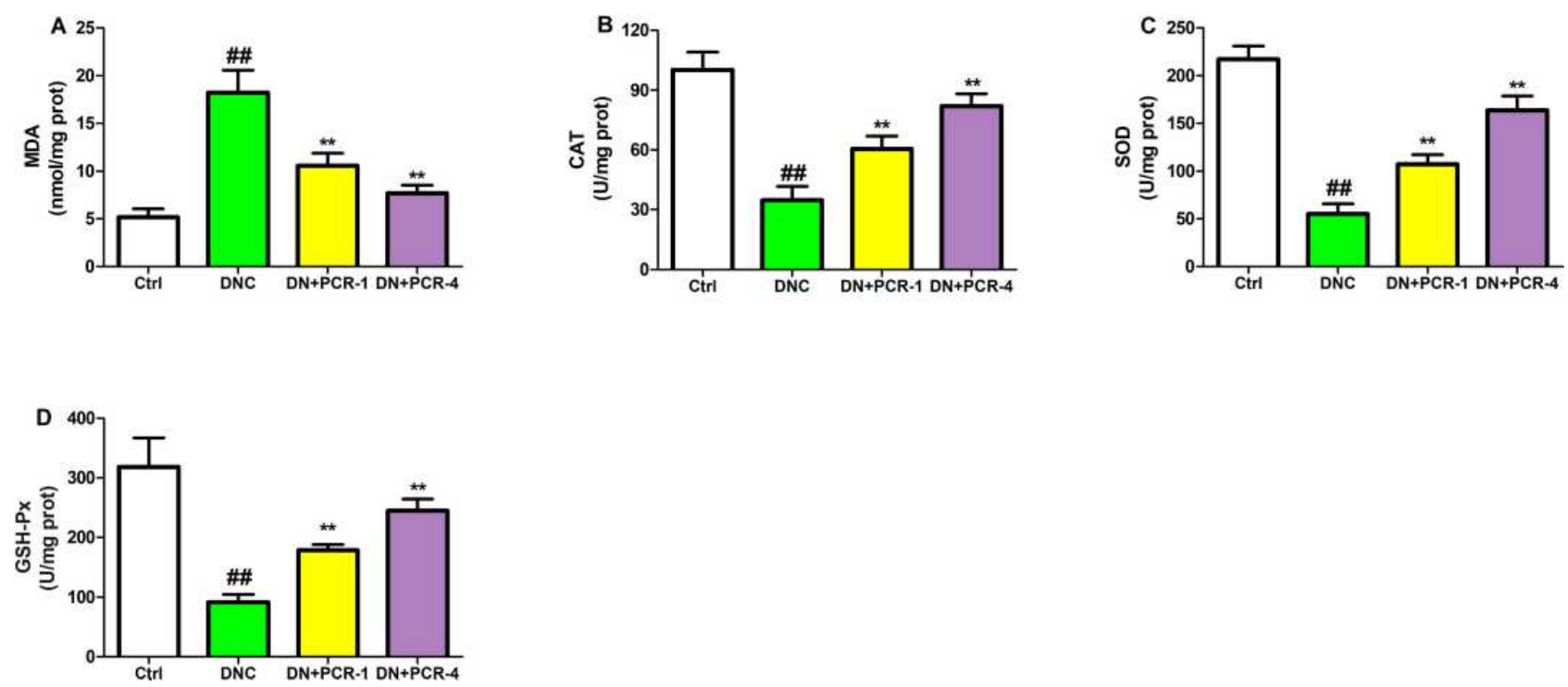

Figure 4 Effect of PCR on renal (A) malondialdehyde, (B) catalase activity, (C) superoxide dismutase activity, (D) glutathione peroxidase activity in DN rats. Data were expressed as the means \pm SD $(n=6)$. Statistical analysis was performed by one-way ANOVA followed by Newman-Keuls post hoc test for multiple comparisons. Notes: ${ }^{\#}$ p 0.05 vs Ctrl group; **p<0.05 vs DNC group.

Abbreviations: Ctrl, control group; DNC, diabetic nephropathy control group; DN+PCR-I, diabetic nephropathy rats treated with PCR at I00mg/kg; DN+PCR-4, diabetic nephropathy rats treated with PCR at $400 \mathrm{mg} / \mathrm{kg}$.

contrast to the results observed for the DN control group, PCR significantly upregulated the altered activities of CAT, SOD and GSH-Px in the kidney of DN rats (Figure 4B-D).

\section{Treatment with PCR Ameliorated Kidney Proinflammatory Cytokines in DN Rats}

As illuminated in Figure 5, the kidney concentrations of proinflammatory cytokines including IL-1 $\beta$, IL-6, TNF- $\alpha$ and TGF- $\beta$ were all significantly increased in the DN control group compared with those of the healthy normal group. Nevertheless, PCR (100 and $400 \mathrm{mg} / \mathrm{kg}$ ) significantly reduced the kidney proinflammatory cytokine levels compared to the DN control group (Figure $5 \mathrm{~A}-\mathrm{D})$.

\section{Treatment with PCR Ameliorated Kidney Histopathological Alterations in DN Rats} As portrayed in Figure 6, the representative kidney histopathological image of the healthy normal control group presented normal glomerular architecture and tubule morphology, with no obvious signs of abnormal 

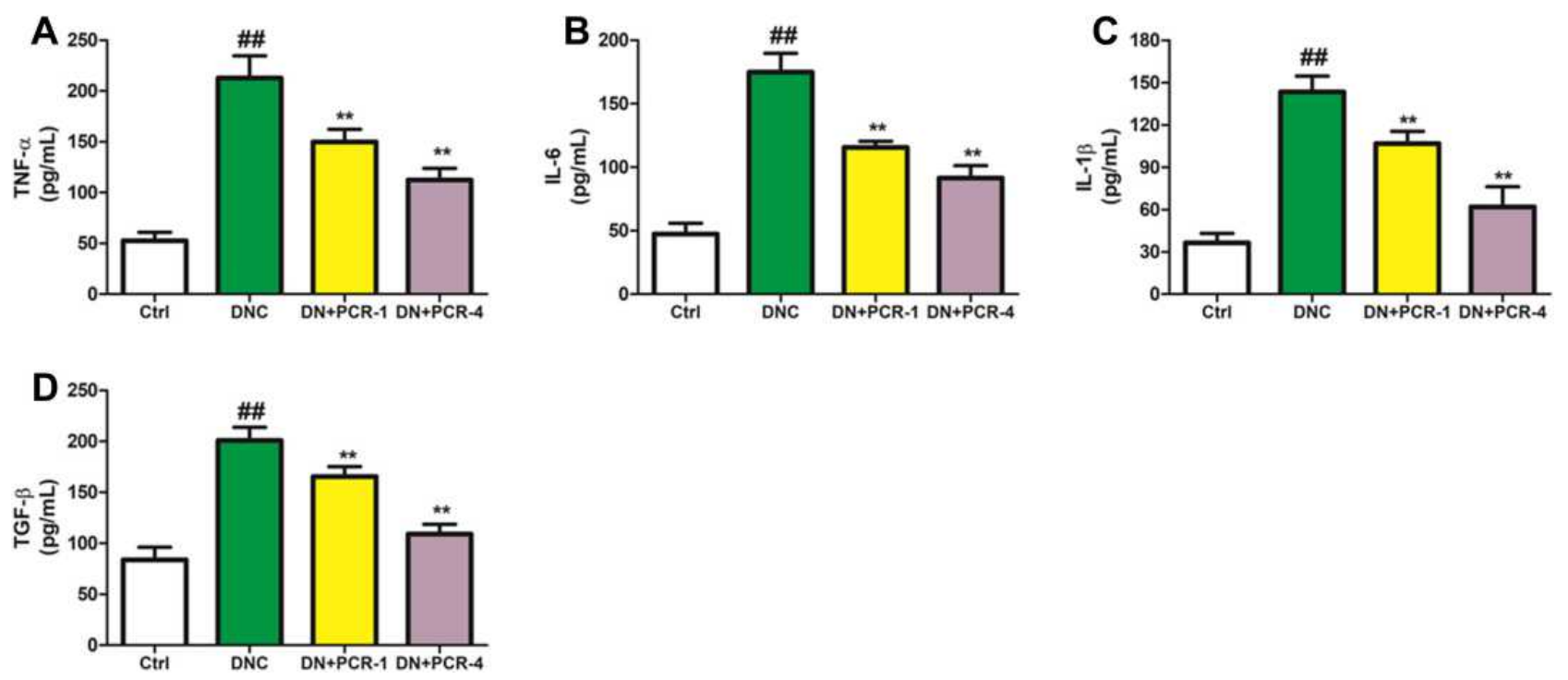

Figure 5 Effect of PCR on renal (A) tumor necrosis factor alpha, (B) interleukin 6, (C) interleukin I beta, (D) transforming growth factor beta in DN rats. Data were expressed as the means \pm SD $(n=6)$. Statistical analysis was performed by one-way ANOVA followed by Newman-Keuls post hoc test for multiple comparisons. Notes: ${ }^{\#}$ p 0.05 vs Ctrl group; $* * p<0.05$ vs DNC group.

Abbreviations: Ctrl, control group; DNC, diabetic nephropathy control group; DN+PCR-I, diabetic nephropathy rats treated with PCR at I00mg/kg; DN+PCR-4, diabetic nephropathy rats treated with PCR at $400 \mathrm{mg} / \mathrm{kg}$.
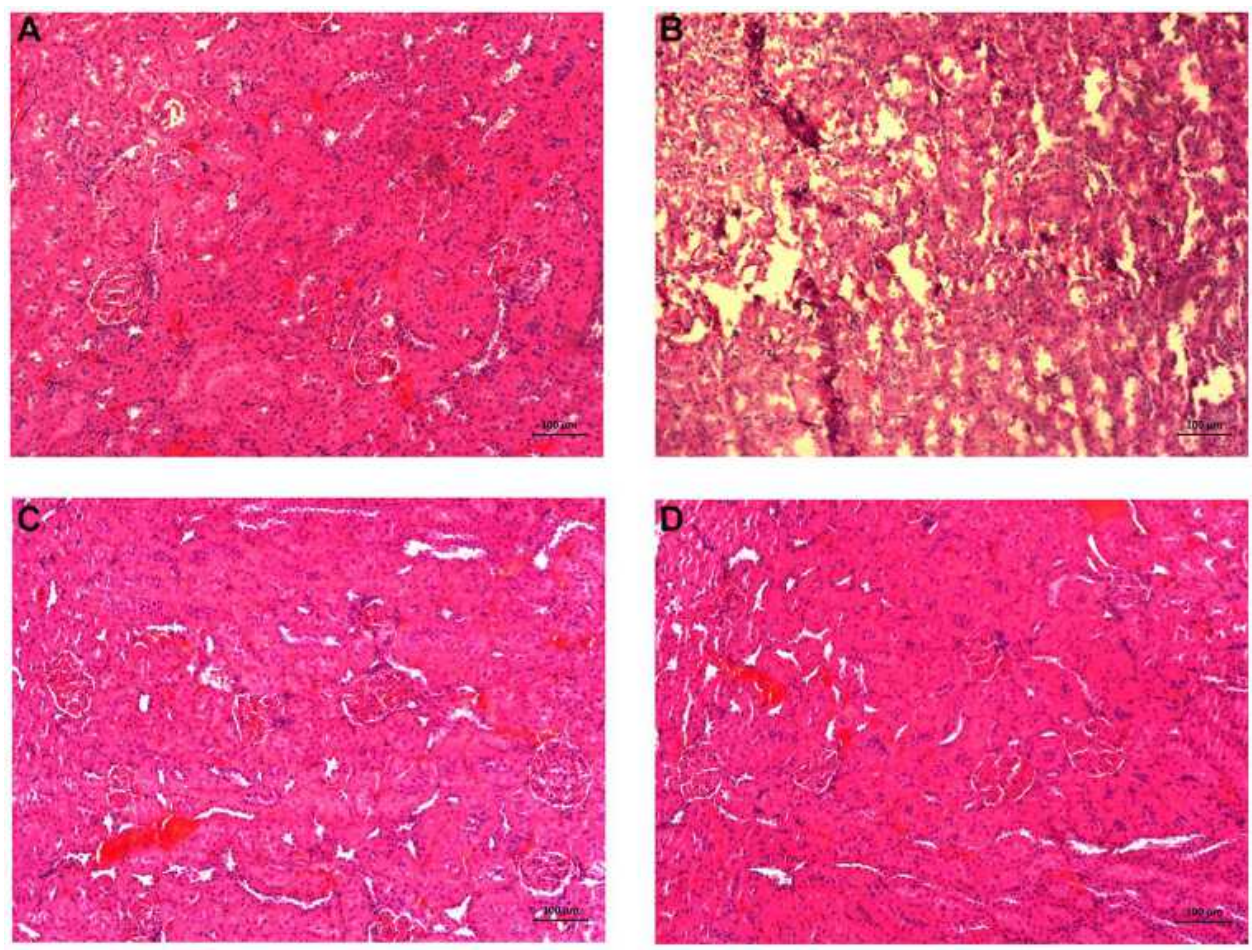

Figure 6 Effect of PCR on histopathological changes in the kidneys of DN rats. Representative histology were conducted using H\&E staining from the experimental groups. (A) Ctrl group, (B) DNC group, (C) DN+PCR-I, (D) DN+PCR-4.

Abbreviations: Ctrl, control group; DNC, diabetic nephropathy control group; DN+PCR-I, diabetic nephropathy rats treated with PCR at I00mg/kg; DN+PCR-4, diabetic nephropathy rats treated with PCR at $400 \mathrm{mg} / \mathrm{kg}$. (Original magnification: $200 \times$, scale bar: $100 \mu \mathrm{m}$ ).

kidney damages (Figure 6A). However, the representative image of the DN control group showed loss of normal glomerular architecture including reduction of glomerular space, detached basement membrane, endothelial cell damage and necrosis (Figure 6B). Notably, PCR could significantly improve these pathological alterations in the DN-treated rats (Figure 6C and D). 
A

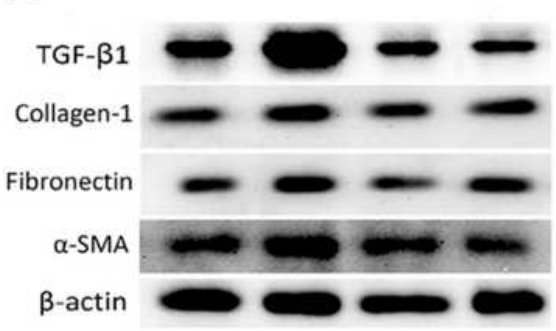

B
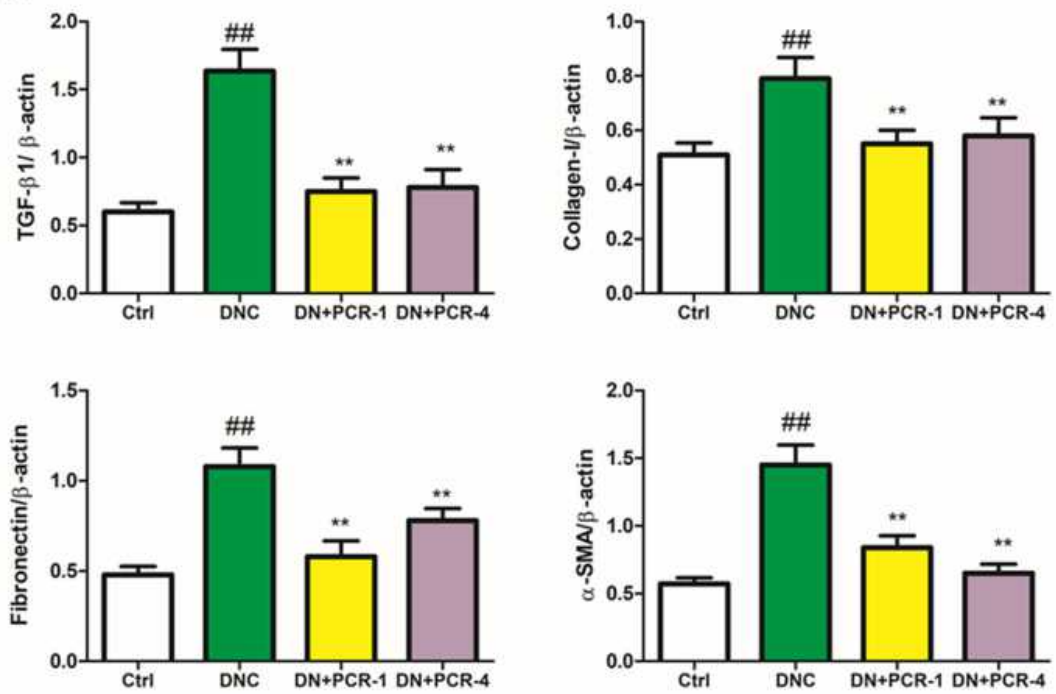

Figure 7 Effect of PCR on renal fibrosis biomarkers in of DN rats. (A) Protein expression of TGF- $\beta$ I, collagen-I, fibronectin and $\alpha$-SMA in DN rats was measured by Western blot analysis and $\beta$-actin serve as the loading control. (B) Quantitative analysis of band intensity using Image) software. Data were expressed as the means \pm SD ( $n$ = 6). Statistical analysis was performed by one-way ANOVA followed by Newman-Keuls post hoc test for multiple comparisons.

Notes: ${ }^{\#} \mathrm{p}<0.05$ vs Ctrl group; ${ }^{* *} \mathrm{p}<0.05$ vs DNC group.

Abbreviations: Ctrl, control group; DNC, diabetic nephropathy control group; DN+PCR-I, diabetic nephropathy rats treated with PCR at I00mg/kg; DN+PCR-4, diabetic nephropathy rats treated with PCR at $400 \mathrm{mg} / \mathrm{kg}$.

\section{Treatment with PCR Ameliorated Kidney Fibrosis in DN Rats}

According to the results shown in Figure 7, the expressions of TGF- $\beta 1$, collagen-1, $\alpha$-SMA and fibronectin proteins were significantly higher in the DN control group when compared to the normal control. Administration of PCR (100 and $400 \mathrm{mg} / \mathrm{kg}$ ) down-regulated the expression of these ECM-related proteins. These results collectively demonstrated that PCR could attenuate renal fibrosis in $\mathrm{DN}$ rats.

\section{Discussion}

In this present study, we evaluated the antidiabetic and nephroprotective effects of PCR extract against high fructose/STZ-induced DN. The results demonstrated that PCR markedly ameliorated DN in DN rats through its nephroprotective properties related to hypoglycemic effects, suppression of renal function markers, oxidative stress and inflammation. PCR extract dramatically suppressed blood glucose concentration, kidney function parameters, proinflammatory cytokines, oxidative stress and improved kidney histopathological alterations. These results clearly suggested that PCR extract protected the progression of DN. Although there are quite a number of therapeutic drugs available to prevent the development of $\mathrm{DN}$, natural products have displayed safer and better therapeutic efficacy, owing to their unique properties including antioxidant, antidiabetic and anti-inflammatory properties.

Diabetic nephropathy is hyperglycemia-induced renal deterioration consistent with glomerular damage, oxidative injury and albuminuria. It is the most common microvascular complication of DM that has become one of the leading causes of end-stage renal disease. ${ }^{27}$ Currently, the clinical therapy for DN mainly targets blood glucose control and blood pressure without effective prevention of DN. However, the pathogenesis of DN is characterized by increased lipid profile, inflammation, oxidative stress, glomerular damage and decline in renal function. ${ }^{28}$ As such, it is imperative to search for effective and safer natural therapy for DN. Unlike the chemical drugs, bioactive macromolecules, such as polysaccharides from natural resources, have multiple biological activities that are beneficial against several diseases. ${ }^{29}$ It is, however, unknown whether the polysaccharide extract from the seaweed Caulerpa racemosa could alleviate DN, and this constituted the principal purpose of this study.

The DN model used in this study was established by high fructose feeding and intraperitoneal injection of STZ followed by 8 weeks of treatment. The American Diabetes Complications Consortium (AMDCC, http://www.amdcc. org) recommended a low-dose of STZ for DN 
establishment, so we adopted STZ at $35 \mathrm{mg} / \mathrm{kg}$ injection to induce DN. In the current study, DN rats showed elevated levels of fasting blood glucose, serum creatinine, BUN and body weight loss. In the untreated group, DN was confirmed with heavy albuminuria and depressed renal functions compared to normal control. Urinary albumin, BUN and serum creatinine are markers for the progression of $\mathrm{DN}$, indicating glomerular lesions that could trigger progressive glomerulosclerosis. ${ }^{30,31}$ Glomerular basement membrane damage and the consequent glomerular filtration barrier dysfunction contribute to renal damage and urinary albumin. ${ }^{32}$ Previous reports have shown albuminuria/proteinuria and decimated renal function in DN as well as characteristic renal lesions including, hypertrophy, tubule thickening, necrosis and epithelial desquamation. $^{27-29,33}$ In consistent with the findings of these aforementioned studies, our study herein also found albuminuria, renal dysfunction, tubule thickening, glomerular hypertrophy and cytoplasm vacuolation in the DN model rats. Furthermore, the significant decrease in body weight and marked increase in the kidney index may be associated with hyperglycemia-induced catabolic derangement, muscle loss and deposition of fats in renal tissues, respectively. ${ }^{34,35}$ DM-induced dyslipidemia was demonstrated by increased levels of TC, TG and LDL-C in this study. Lipid metabolism is one of the pathways impaired in DM and usually contributes to DN development. ${ }^{28}$ Studies in diabetic patients revealed the adverse effect of dyslipidemia on glomeruli integrity and DN development. ${ }^{36,37}$ Of note, the treatment of $\mathrm{DN}$ rats with PCR reduced the fasting blood glucose levels, increased body weight and decreased the kidney/body weight ratio (Figure 1). In addition, PCR prominently reduced the levels of serum creatinine, BUN, urinary albumin and lipid levels in the PCR-treated DN group compared to the untreated DN group (Figure 2). In earlier studies, polysaccharides from natural sources have been associated with beneficial pharmacological activities, including antidiabetic and nephroprotective effects. ${ }^{38-40}$ For example, polysaccharides from medicinal plants, pumpkin, sea cucumber and mushroom improve insulin sensitivity, alleviate glucose transporters and DM complications. ${ }^{39,41-43}$ In consistent with the antidiabetic effect of PCR herein, the polysaccharides from Turbinaria ornata and Sargassum crassifolium seaweeds lowered blood glucose and improved body weight in DM rats. ${ }^{44}$ The study of BelHadj et al indicated that polysaccharides could protect the kidney function via reduced levels of creatinine, BUN and urea which was in agreement with our findings. ${ }^{45}$

Chronic hyperglycemia provokes glucose toxicity in several tissues and organs via oxidative stress. Oxidative stress, which results from excess free radical production, is strongly implicated in DN as impaired antioxidant defence systems. ${ }^{30,34,46}$ In an attempt to explore the possible antioxidant efficacy of natural agents, previous studies assessed antioxidant enzymes and lipid peroxidation in target organs. Similarly, we evaluated renal SOD, CAT, and GSH-Px activities, as well as MDA in the current investigation. It was strikingly observed that DN aggravated MDA level (Figure $4 \mathrm{~A}$ ) as a consequence of the concomitant decrease in renal SOD, CAT and GSH-Px activities (Figure 4B-D) following accumulating published evidences. ${ }^{28,32,47}$ Existing literatures indicated that impairment of renal antioxidant homeostasis is a critical factor in the development and progression of DN. Reactive oxygen species (ROS) ensuing from uncontrolled hyperglycemia attacks renal cell membrane, causing oxidative damage and lipid peroxidation. ${ }^{48}$ However, certain studies have suggested that excess ROS could induce increased expression of cellular antioxidant machinery, ${ }^{49,50}$ however, this compensatory mechanism may be insufficient to counteract the consumption of antioxidant apparatus. As such, the excessive production of renal ROS could have overwhelmed the counteracting effects of SOD, CAT, and GSH-Px leading to their reduced activities in this study. As a consequence, peroxidative damage in the kidney contributed to the enhanced formation of MDA observed in this study. Interestingly, the administration of PCR was found to prominently increase the activities of SOD, CAT and GSH-Px as well as reduced the levels of MDA. In vivo animal studies have suggested the antioxidant effect of polysaccharides on several fungi, plants and seaweeds. ${ }^{29,38,51}$ Polysaccharides from Grifola frondosa and Salvia miltiorrhiza Bunge attenuated oxidative damage and restored the activities of SOD, CAT, GSH-Px and decreased MDA levels. ${ }^{7,52}$ Thus, the antioxidant effect of PCR may be associated with its ability to inhibit ROS formation and enhanced the activities of SOD, CAT and GSH-Px in the kidney. ${ }^{51}$

Increasing volume of evidence has strongly suggested that oxidative stress is a prime trigger for pro-inflammatory cascades. $^{32,53,54}$ In fact, the crosstalks between oxidative stress and inflammatory response are two linked pathways in DN pathophysiology, which are considered to be a major hallmark of DN. On the one hand, however, the renal histopathology of the untreated DN rats revealed vacuolated cytoplasm, tubule basement thickening and glomerular 
hypertrophy. In particular, tubule basement thickening and glomerular hypertrophy are peculiar histological features of DN. ${ }^{32,34}$ Our study portrayed a significant increase in TNF$\alpha$, IL-1 $\beta$, IL- 6 and TGF- $\beta$ levels in the kidneys of DN rats (Figure 5). The increased expression of TGF- $\beta$ has been previously linked to renal hypertrophy. ${ }^{30}$ In agreement with our findings, previous studies have also reported prominent increase in renal TNF- $\alpha$, TGF- $\beta$, IL- $1 \beta$ and IL- 6 levels in DN models. ${ }^{28,47}$ In contrast, PCR administration resisted the pro-inflammatory mechanism evident by a dose dependent reduction in TNF- $\alpha$, TGF- $\beta$, IL- $1 \beta$ and IL- 6 levels.

The prominent role that TGF- $\beta 1$ plays in extracellular matrix (ECM) deposition and evoking renal fibrosis cannot be overemphasized. Accumulating evidence has stressed the importance of TGF- $\beta$ signaling pathway in the development and progression of DN. The extent of renal fibrosis in DN largely depends on the accumulation of ECM protein, the mesangial cell matrix deposition and thickening of the glomeruli basement membrane. ${ }^{55,56}$ In addition, TGF- $\beta$ pathway has been proposed to induce renal fibrosis through the expression of $\alpha$-SMA. ${ }^{57,58}$ Consistent with $\mathrm{DN}$, the results obtained from this study demonstrated that the expression of TGF- $\beta 1, \alpha-S M A$, collagen-1and and fibronectin proteins were significantly upregulated in $\mathrm{DN}$ rats in contrast to the normal control rats and the administration of PCR displayed significant regulatory effects on the expression of these fibrosis-related biomarkers (Figure 7). These results strongly suggested that PCR could improve renal fibrosis in $\mathrm{DN}$ model rats.

\section{Conclusion}

In conclusion, our study findings strongly suggested that PCR possess antioxidant and anti-inflammatory properties that can be applied to prevent progression of diabetic nephropathy. We observed that PCR extract significantly reduced blood glucose concentration, proinflammatory cytokines, oxidative stress, dyslipidemia, renal function biomarkers and improved kidney histopathological damages. PCR also increased renal antioxidant capacity by inducing SOD, CAT, and GSH-Px levels in DN rats. While these findings and that of other researchers await clinical confirmation, further studies need to explore molecular mechanisms for PCR efficacy related to apoptosis, ferroptosis, nuclear transcription factors, phosphatidylinositol-3-kinase/kinase B (PI3K/Akt), peroxisome proliferator-activated receptor- $\gamma$ (PPAR- $\gamma)$ genes.

\section{Disclosure}

The authors declared no conflicts of interest for this work.

\section{References}

1. International Diabetes Federation. IDF Diabetes Atlas. Ninth edition. International Diabetes Federation; 2019: 9.

2. Makinde EA, Radenahmad N, Zaman RU, Olatunji OJ. Fatty acids and sterol rich stem back extract of Shorea roxburghii attenuates hyperglycemia, hyperlipidemia, and oxidative stress in diabetic rats. Eur J Lipid Sci Technol. 2020;122:2000151. doi:10.1002/ ejlt.202000151

3. Gerber PA, Rutter GA. The role of oxidative stress and hypoxia in pancreatic beta-cell dysfunction in diabetes mellitus. Antioxidants Redox Signal. 2017;26:501-518. doi:10.1089/ars.2016.6755

4. Andrade C, Gomes NGM, Duangsrisai S, Andrade PB, Pereira DM, Valentão P. Medicinal plants utilized in Thai Traditional Medicine for diabetes treatment: ethnobotanical surveys, scientific evidence and phytochemicals. J Ethnopharmacol. 2020;263:113177.

5. Stanton RC. Clinical challenges in diagnosis and management of diabetic kidney disease. Am J Kidney Dis. 2014;63:S3-S21. doi:10.1053/j.ajkd.2013.10.050

6. Tervaert TWC, Mooyaart AL, Amann K, et al. Pathologic classification of diabetic nephropathy. J Am Soc Nephrol. 2010;21:556-563. doi:10.1681/ASN.2010010010

7. Porrini E, Ruggenenti P, Mogensen CE, et al. Non-proteinuric pathways in loss of renal function in patients with type 2 diabetes. Lancet Diabetes Endocrinol. 2015;3:382-391. doi:10.1016/S2213-8587(15) 00094-7

8. Xie R, Zhang H, Wang XZ, et al. The protective effect of betulinic acid (BA) diabetic nephropathy on streptozotocin (STZ)-induced diabetic rats. Food Funct. 2017;8:299-306. doi:10.1039/ C6FO01601D

9. Giacco F, Brownlee M, Schmidt AM. Oxidative stress and diabetic complications. Circ Res. 2010;107:1058-1070. doi:10.1161/ CIRCRESAHA.110.223545

10. Elsherbiny NM, Al-Gayyar MMH, Abd El Galil KH. Nephroprotective role of dipyridamole in diabetic nephropathy: effect on inflammation and apoptosis. Life Sci. 2015;143:8-17. doi:10.1016/ j.1fs. 2015.10 .026

11. Ni Z, Guo L, Liu F, Olatunji OJ, Yin M. Allium tuberosum alleviates diabetic nephropathy by suppressing hyperglycemia-induced oxidative stress and inflammation in high fat diet/streptozotocin treated rats. Biomed Pharmacother. 2019;112:108678. doi:10.1016/j. biopha.2019.108678

12. Rengasamy KR, Mahomoodally MF, Aumeeruddy MZ, Zengin G, Xiao J, Kim DH. Bioactive compounds in seaweeds: an overview of their biological properties and safety. Food Chem Toxicol. 2020;135:111013. doi:10.1016/j.fct.2019.111013

13. Cardoso SM, Pereira OR, Seca AM, Pinto DC, Silva AM. Seaweeds as preventive agents for cardiovascular diseases: from nutrients to functional foods. Mar Drugs. 2015;13:6838-6865. doi:10.3390/ md13116838

14. Yap WF, Tay V, Tan SH, Yow YY, Chew J. Decoding antioxidant and antibacterial potentials of Malaysian green seaweeds: Caulerpa racemosa and Caulerpa lentillifera. Antibiotics (Basel). 2019;8:3. doi:10.3390/antibiotics 8030152

15. Yangthong M, Hutadilok-Towatana N, Phromkunthong W. Antioxidant activities of four edible seaweeds from the southern coast of Thailand. Plant Foods Hum Nutr. 2009;64:218-223. doi:10.1007/s11130-009-0127-y

16. Hao H, Han Y, Yang L, et al. Structural characterization and immunostimulatory activity of a novel polysaccharide from green alga Caulerpa racemosa var peltata. Int $J$ Biol Macromol. 2019;134:891-900. doi:10.1016/j.ijbiomac.2019.05.084 
17. Ribeiro NA, Abreu TM, Chaves HV, et al. Sulfated polysaccharides isolated from the green seaweed Caulerpa racemosa plays antinociceptive and anti-inflammatory activities in a way dependent on HO-1 pathway activation. Inflamm Res. 2014;63:569-580. doi:10.1007/ s00011-014-0728-2

18. Ribeiro NA, Chaves HV, da Conceição Rivanor RL, et al. Sulfated polysaccharide from the green marine algae Caulerpa racemosa reduces experimental pain in the rat temporomandibular joint. Int $\mathrm{J}$ Biol Macromol. 2020;150:253-260. doi:10.1016/j.ijbiomac.2020.01.272

19. Olatunji OJ, Feng Y, Olatunji OO, et al. Polysaccharides purified from Cordyceps cicadae protects PC12 cells against glutamate-induced oxidative damage. Carbohydr Polym. 2016;153:187-195. doi:10.1016/j.carbpol.2016.06.108

20. Yuan Y, Zhou J, Zheng Y, et al. Beneficial effects of polysaccharide-rich extracts from Apocynum venetum leaves on hypoglycemic and gut microbiota in type 2 diabetic mice. Biomed Pharmacother. 2020;127:110182. doi:10.1016/j.biopha.2020.110182

21. Kale OE, Akinpelu OB, Bakare AA, et al. Five traditional Nigerian polyherbal remedies protect against high fructose fed, Streptozotocin-induced type 2 diabetes in male Wistar rats. $B M C$ Complement Altern Med. 2018;18:160. doi:10.1186/s12906-0182225-6

22. Obafemi TO, Olaleye MT, Akinmoladun AC. Antidiabetic property of miracle fruit plant (Synsepalum dulcificum Shumach. \& Thonn. Daniell) leaf extracts in fructose-fed streptozotocin-injected rats via anti-inflammatory activity and inhibition of carbohydrate metabolizing enzymes. J Ethnopharmacol. 2019;244:112124. doi:10.1016/j. jep.2019.112124

23. Qudus B Aroyehun A, Abdul Razak S, Palaniveloo K. Bioprospecting cultivated tropical green algae, Caulerpa racemosa (Forsskal) J. Agardh: a perspective on nutritional properties, antioxidative capacity and anti-diabetic potential. Foods. 2020;9:1313. doi:10.3390/foods9091313

24. Zhang Q, Olatunji OJ, Chen H, Tola AJ, Oluwaniyi OO. Evaluation of the anti-diabetic activity of polysaccharide from Cordyceps cicadae in experimental diabetic rats. Chem Biodivers. 2018;15: e1800219. doi:10.1002/cbdv.201800219

25. Mestry SN, Dhodi JB, Kumbhar SB, Juvekar AR. Attenuation of diabetic nephropathy in streptozotocin-induced diabetic rats by Punica granatum Linn. leaves extract. J Tradit Complement Med. 2016;7:273-280. doi:10.1016/j.jtcme.2016.06.008

26. Yang J, Dong H, Wang Y, et al. Cordyceps cicadae polysaccharides ameliorated renal interstitial fibrosis in diabetic nephropathy rats by repressing inflammation and modulating gut microbiota dysbiosis. Int J Biol Macromol. 2020;163:442-456. doi:10.1016/j.ijbiomac.20 20.06.153

27. Yang K, Bai Y, Yu N, et al. Huidouba improved podocyte injury by down regulating Nox4 expression in rats with diabetic nephropathy. Front Pharmacol. 2020;11:587995. doi:10.3389/fphar.2020.587995

28. Cui Y, Shi Y, Bao Y, Wang S, Hua Q, Liu Y. Zingerone attenuates diabetic nephropathy through inhibition of nicotinamide adenine dinucleotide phosphate oxidase 4. Biomed Pharmacother. 2018;99:422-430. doi:10.1016/j.biopha.2018.01.051

29. Shen M, Chen X, Huang L, Yu Q, Chen Y, Xie J. Sulfated Mesona chinensis Benth polysaccharide enhance the immunomodulatory activities of cyclophosphamide-treated mice. $J$ Funct Food. 2021;76:104321. doi:10.1016/j.jff.2020.104321

30. Alaofi AL. Sinapic acid ameliorates the progression of streptozotocin (STZ)-induced diabetic nephropathy in rats via Nrf2/HO-1 mediated pathways. Front Pharmacol. 2020;11:1119. doi:10.3389/fphar.2020. 01119

31. Tucker PS, Scanlan AT, Dalbo VJ. Chronic kidney disease influences multiple systems: describing the relationship between oxidative stress, inflammation, kidney damage, and concomitant disease. Oxid Med Cell Longev. 2015;2015:806358. doi:10.1155/2015/806358
32. An X, Zhang L, Yuan Y, et al. Hyperoside pre-treatment prevents glomerular basement membrane damage in diabetic nephropathy by inhibiting podocyte heparanase expression. Sci Rep. 2017;7:6413. doi:10.1038/s41598-017-06844-2

33. Oh SW, Kim S, Na KY, et al. Clinical implications of pathologic diagnosis and classification for diabetic nephropathy. Diabetes Res Clin Pract. 2012;97:418-424. doi:10.1016/j.diabres.2012.03.016

34. Li F, Chen Y, Li Y, Huang M, Zhao W. Geniposide alleviates diabetic

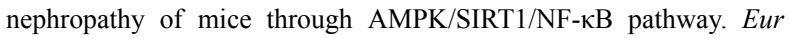
J Pharmacol. 2020;886:173449. doi:10.1016/j.ejphar.2020.173449

35. Qiao Y, Gao K, Wang Y, Wang X, Cui B. Resveratrol ameliorates diabetic nephropathy in rats through negative regulation of the $\mathrm{p} 38$ MAPK/TGF-beta 1 pathway. Exp Ther Med. 2017;13:3223-3230. doi:10.3892/etm.2017.4420

36. Palazhy S, Viswanathan V. Lipid abnormalities in type 2 diabetes mellitus patients with overt nephropathy. Diabetes Metab J. 2017;41:128-134. doi:10.4093/dmj.2017.41.2.128

37. Kawanami D, Matoba K, Utsunomiya K. Dyslipidemia in diabetic nephropathy. Ren Replace Ther. 2016;2:1-9. doi:10.1186/s41100016-0028-0

38. Ganesan K, Xu B. Anti-diabetic effects and mechanisms of dietary polysaccharides. Molecules. 2019;24:2556-2583. doi:10.3390/ molecules24142556

39. Chen Y, Liu Y, Sarker MMR, et al. Structural characterization and antidiabetic potential of a novel heteropolysaccharide from Grifola frondosa via IRS1/PI3K-JNK signalling pathways. Carbohydr Polym. 2018;198:452-461. doi:10.1016/j.carbpol.2018.06.077

40. Tang HL, Chen C, Wang SK, Sun GJ. Biochemical analysis and hypoglycemic activity of a polysaccharide isolated from the fruit of Lycium barbarum L. Int J Biol Macromol. 2015;77:235-242. doi:10.1016/j.ijbiomac.2015.03.026

41. Yang ZM, Wang Y, Chen SY. Astragalus polysaccharide alleviates type 2 diabetic rats by reversing the glucose transporters and sweet taste receptors/GLP-1/GLP-1 receptor signaling pathways in the intestine-pancreatic axis. $J$ Funct Foods. 2021;76:104310. doi:10.1016/j.jff.2020.104310

42. Liu C, Song J, Teng M, et al. Antidiabetic and antinephritic activities of aqueous extract of Cordyceps militaris fruit body in diet-streptozotocin-induced diabetic Sprague Dawley rats. Oxid Med Cell Longev. 2016;2016:1-11.

43. Zhao T, Mao GH, Zhang M, et al. Anti-diabetic effects of polysaccharides from ethanol-insoluble residue of Schisandra chinensis (Turcz.) Baill on alloxan-induced diabetic mice. Chem Res Chin Univ. 2012;29:99-102. doi:10.1007/s40242-012-2218-9

44. Husni A. Therapeutic potential of seaweed polysaccharides for diabetes mellitus. Seaweed biomaterials. IntechOpen. 2018. doi:10. 5772/intechopen. 76570

45. BelHadj S, Hentati O, Abdelfattah E, Hamden K. Inhibitory activities of Ulva lactuca polysaccharides on digestive enzymes related to diabetes and obesity. Arch Physiol Biochem. 2013;119:81-87. doi: $10.3109 / 13813455.2013 .775159$

46. Zhang H, Shi T, Wang J, Li R, Tang W. Protective effect of penta-acetyl geniposide on acute liver injury induced by D-galactosamine in mice. Br J Pharmacol Toxicol. 2013;4:256-261. doi:10.19026/bjpt.4.5409

47. Singh B, Kumar A, Singh H, et al. Zingerone produces antidiabetic effects and attenuates diabetic nephropathy by reducing oxidative stress and overexpression of NF- $\kappa \mathrm{B}, \mathrm{TNF}-\alpha$, and COX-2 proteins in rats. J Funct Foods. 2020;74:104199. doi:10.1016/j.jff.2020.104199

48. Kotsafti A, Scarpa M, Castagliuolo I, Scarpa M. Reactive oxygen species and antitumor immunity-from surveillance to evasion. Cancers. 2020;12:1748-1764. doi:10.3390/cancers12071748

49. Miyazono Y, Gao F, Horie T. Oxidative stress contributes to methotrexate- induced small intestinal toxicity in rats. Scand $J$ Gastroenterol. 2004;39:1119-1127. doi:10.1080/0036552041000 3605 
50. Uzar E, Koyuncuoglu HR, Uz E, et al. The activities of antioxidant enzymes and the level malondialdehyde in cerebellum of rats subjected to methotrexate: protective effect of caffeic acid phenetyl ester. Mol Cell Biochem. 2006;291:63-68. doi:10.1007/s11010-006-9196-5

51. Kim KJ, Lee BY. Fucoidan from the sporophyll of Undaria pinnatifida suppresses adipocyte differentiation by inhibition of inflammation-related cytokines in 3T3-L1 cells. Nutr Res. 2012;32:439-447. doi:10.1016/j.nutres.2012.04.003

52. Kou L, Du M, Liu P, et al. Anti-diabetic and anti-nephritic activities of Grifola frondosa mycelium polysaccharides in diet-streptozotocininduced diabetic rats via modulation on oxidative stress. Appl Biochem Biotechnol. 2018;187:310-322. doi:10.1007/s12010-0182803-6

53. Famurewa AC, Edeogu CO, Offor FI, Besong EE, Akunna GG, Maduagwuna EK. Downregulation of redox imbalance and iNOS/ $\mathrm{NF}-\mathrm{KB}$ /caspase-3 signalling with zinc supplementation prevents urotoxicity of cyclophosphamide-induced hemorrhagic cystitis in rats. Life Sci. 2021;266:118913. doi:10.1016/j.1fs.2020.118913
54. Zhao J, Liu L, Li X, et al. Neuroprotective effects of an Nrf2 agonist on high glucose-induced damage in HT22 cells. Biol Res. 2019;52:53-63. doi:10.1186/s40659-019-0258-z

55. Lu Q, Zuo WZ, Ji XJ, et al. Ethanolic Ginkgo biloba leaf extract prevents renal fibrosis through Akt/mTOR signaling in diabetic nephropathy. Phytomedicine. 2015;22:1071-1078. doi:10.1016/j. phymed.2015.08.010

56. Ponnusamy M, Zhou X, Yan Y, et al. Blocking sirtuin 1 and 2 inhibits renal interstitial fibroblast activation and attenuates renal interstitial fibrosis in obstructive nephropathy. $J$ Pharmacol Exp Ther. 2014;350:243-256. doi:10.1124/jpet.113.212076

57. Gu L-Y, Tang H-T, Xu Z-X. Huangkui capsule in combination with metformin ameliorates diabetic nephropathy via the Klotho/TGF- $\beta 1$ / p38MAPK signaling pathway. J Ethnopharmacol. 2020;113548. doi:10.1016/j.jep.2020.113548

58. Kundu A, Dey P, Sarkar P, et al. Protective effects of Croton hookeri on streptozotocin-induced diabetic nephropathy. Food Chem Toxicol. 2020;135:110873. doi:10.1016/j.fct.2019.110873

Diabetes, Metabolic Syndrome and Obesity: Targets and Therapy

Dovepress

\section{Publish your work in this journal}

Diabetes, Metabolic Syndrome and Obesity: Targets and Therapy is an international, peer-reviewed open-access journal committed to the rapid publication of the latest laboratory and clinical findings in the fields of diabetes, metabolic syndrome and obesity research. Original research, review, case reports, hypothesis formation, expert opinion and commentaries are all considered for publication. The manuscript management system is completely online and includes a very quick and fair peer-review system, which is all easy to use. Visit http://www.dovepress.com/testimonials.php to read real quotes from published authors.

Submit your manuscript here: https://www.dovepress.com/diabetes-metabolic-syndrome-and-obesity-targets-and-therapy-journal 